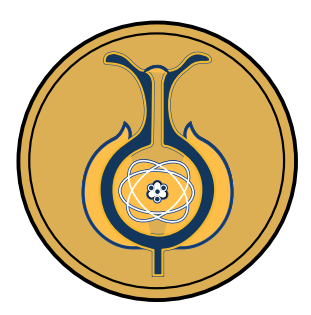

\title{
Fluorescencia, reflectancia y respuesta fisiológica al estrés hídrico en plántulas de Gmelina arborea Roxb
}

Fluorescence, reflectance, and physiological response to water stress in Gmelina arborea Roxb seedlings

Fluorescência, reflectância e resposta fisiológica ao estresse hídrico em plântulas de Gmelina arborea Roxb

Juan Carlos Valverde ${ }^{1,2} \cdot$ Dagoberto Arias-Aguilar $^{2} \cdot$ Ernesto Montero-Zeledón $^{3} \cdot$ Dionisio Gutiérrez-Fallas $^{3}$

Received: Jul/20/2020 • Accepted: Aug/22/2020 • Published: Jan/31/2021

\section{Resumen}

Se realizó una caracterización de la fluorescencia, reflectancia y respuesta fisiológica al estrés hídrico en plántulas de Gmelna arborea Roxb. Para lo cual, se desarrolló un experimento durante 31 días con dos tratamientos: plantas testigo (se aplicó un régimen hídrico normal) y plantas en condición de estrés hídrico (a las que se les limitó en su totalidad el agua por el periodo del experimento). Para ambos métodos se implementaron clones de $G$. arborea y se evaluaron cada siete días hasta el final del experimento, se analizó la fotosíntesis, conductancia estomática, turgencia foliar, contenido relativo de clorofila, fluorescencia y reflectancia. Los resultados mostraron que la biomasa en las plantas con estrés se redujo en un 26,9\% siendo la cobertura foliar la que mostró mayor afectación (disminución del $29,1 \%$ ), la reducción de la fotosíntesis fue del $60,1 \%\left(5,8 \mu \mathrm{mol} \mathrm{m} \mathrm{m}^{-2} \mathrm{~s}^{-1}\right)$ con respecto a la planta testigo $\left(14,6 \mu \mathrm{mol} \mathrm{m}^{-2} \mathrm{~s}^{-1}\right)$. Para las variables conductancia estomática, turgencia y contenido relativo de clorofila el comportamiento fue de disminuir sus valores de un 20 a $60 \%$ conforme el periodo de estrés se incrementó. Con respecto a la fluorescencia se determinaron dos patrones: de los 400 a $550 \mathrm{~nm}$ la fluorescencia se incrementó en las plantas estresadas, mientras que a partir de los 600 a $725 \mathrm{~nm}$ fueron las plantas testigo las que presentaron mayor excitación, en ambos rangos se presentaron valores máximos en los puntos correspondientes a clorofila a y b. Finalmente, la reflectancia mostró diferenciación entre los 460 y $770 \mathrm{~nm}$ siendo las plantas en condiciones de estrés las que presentaron una reflectancia mayor. El patrón obtenido de degradación fisiológica de las plantas estresadas se debe a la pérdida de agua del organismo que incide en cierre de estomas, aumento de producción de ácido abscísico, disminución de la síntesis de pared celular y limitaciones en la síntesis de proteínas.

Palabras clave: Fisiología forestal; estrés hídrico; Gmelina arborea; degradación fisiológica.

\footnotetext{
Juan Carlos Valverde, $\$ jcvalverde@tec.ac.cr, Orcid ID: (D https://orcid.org/0000-0002-3181-1346

Dagoberto Arias-Aguilar, $\$ darias@tec.ac.cr, Orcid ID: (1D https://orcid.org/0000-0002-3056-9172

Ernesto Montero-Zeledón, \ emontero@tec.ac.cr, Orcid ID: (10 https://orcid.org/0000-0002-4545-5805

Dionisio Gutierrez-Fallas, $\square$ dgutierrez@tec.ac.cr, Orcid ID: (D https://orcid.org/0000-0001-7190-8858

1 Facultad de Ciencias Forestales, Universidad de Concepción. Concepción, Chile.

2 Laboratorio de Ecofisiología Forestal y Aplicaciones Ecosistémicas (ECOPLANT), Tecnológico de Costa Rica. Cartago, Costa Rica.

3 Escuela de Física, Tecnológico de Costa Rica. Cartago, Costa Rica.
} 


\section{Abstract}

Fluorescence, reflectance, and physiological response to water stress were analyzed in Gmelina arborea Roxb seedlings. The experiment was conducted for 31 days with two treatments: control plants (a normal water regime was applied) and plants under water stress conditions (water was completely limited for these plants during the extent of the experiment). For both treatments, $G$. arborea clones were used and evaluated every seven days until the end of the experiment. Photosynthesis, stomatal conductance, leaf turgor, relative chlorophyll content, fluorescence, and reflectance were analyzed. Results showed that the biomass in plants under stress was reduced by $26.9 \%$, being the leaf coverage the one with the greatest affectation (29.1\% decrease), while photosynthesis was reduced by $60.1 \%\left(5.8 \mu \mathrm{mol} \mathrm{m}^{-2} \mathrm{~s}^{-1}\right)$ compared to the control plant $\left(14.6 \mu \mathrm{mol} \mathrm{m} \mathrm{m}^{-2} \mathrm{~s}^{-1}\right)$. For the variables stomatal conductance, turgor, and relative chlorophyll content, the behavior was to decrease values from 20 to $60 \%$ as the stress period increased. With respect to fluorescence, two standards were determined: from 400 to $550 \mathrm{~nm}$, fluorescence increased in stressed plants, while from 600 to $725 \mathrm{~nm}$, control plants showed the highest excitation. In both ranges, maximum values were presented at the points corresponding to chlorophyll a and b. Finally, reflectance showed differences between 460 and $770 \mathrm{~nm}$, being the plants under stress conditions the ones showing the highest reflectance. The physiological degradation pattern obtained in the stressed plants is due to the organism's water loss resulting in stomatal closure, increased production of abscisic acid, decreased cell wall synthesis, and limitations in protein synthesis.

Keywords: Forest physiology; water stress; Gmelina arborea; physiological degradation

\section{Resumo}

Foi realizada uma caracterização da fluorescência, reflectância e resposta fisiológica ao estresse hídrico em plântulas de Gmelina arborea Roxb. Para o qual, se desenvolveu um experimento por 31 dias com dois tratamentos: plantas de controle (aplicou-se um regime hídrico normal) e plantas sob condição de estresse hídrico (às quais se limitou a água completamente pelo período do experimento). Para ambos os métodos foram implementados clones de $G$. arborea e avaliados a cada sete dias até o final do experimento, foram analisadas a fotossínteses, a condutância estomática, a turgência foliar, o teor relativo de clorofila, fluorescência e reflectância. Os resultados mostraram que a biomassa nas plantas sob estresse reduziu $26,9 \%$ sendo a cobertura foliar a que mostrou maior detrimento (diminuição de 29,1 \%), a redução da fotossíntese foi de $60,1 \%\left(5,8 \mu \mathrm{mol} \mathrm{m} \mathrm{m}^{-2} \mathrm{~s}^{-1}\right)$ em relação à planta controle $\left(14,6 \mu \mathrm{mol} \mathrm{m} \mathrm{m}^{-2} \mathrm{~s}^{-1}\right)$. Para as variáveis condutância estomática, turgência e teor relativo de clorofila, o comportamento foi diminuir seus valores de 20 a $60 \%$ conforme aumentava o período de estresse. No que diz respeito à fluorescência, foram determinados dois padrões: dos 400 a $550 \mathrm{~nm}$, em que a fluorescência aumentou nas plantas estressadas e, a partir dos 600 a $725 \mathrm{~nm}$, em que foram as plantas de controle as que apresentaram maior excitação; em ambas as faixas foram apresentados valores máximos nos pontos correspondentes à clorofila a e b. Finalmente, a reflectância mostrou diferenciação entre os 460 e 770 nm, sendo as plantas sob condições de estresse as que apresentaram uma reflectância maior. 0 padrão obtido de degradação fisiológica das plantas estressadas deve-se à perda de água do organismo que incide no fechamento dos estômatos, aumento de produção de ácido abscísico, diminuição da síntese da parede celular e limitações na síntese de proteínas.

Palavras-chave: fisiologia florestal; estresse hídrico; Gmelina arborea; degradaç̧ão fisiológica. 


\section{INTRODUCCIÓN}

El estrés fisiológico en plantas es la respuesta a la exposición de condiciones adversas que alteran el desarrollo de la planta, tales como variaciones de temperatura, presión atmosférica, régimen hídrico, salinidad o una combinación de los factores anteriores (Valverde y Arias, 2020). Estos pueden generar daños temporales o permanentes en el organismo: afectan el crecimiento, procesos reproductivos y sobrevivencia de la planta (Beyer et al., 2014; Paulus et al., 2014). En el caso específico del estrés hídrico, generalmente se refiere al déficit de agua por periodos prolongados (Gautier et al., 2000), aspecto que genera una reacción inmediata en la planta: reducción de la tasa de crecimiento producto de la reducción del volumen celular y aumento de solutos orgánicos que inciden en daños mecánicos en la planta (Farooq et al., 2009; Moreno, 2009). Además, combinado con un desbalance entre la transpiración y absorción de agua, se generan daños físicos en el organismo (Ortuño et al., 2006; Varone et al., 2012).

El estrés hídrico puede generar tres niveles de afectación al desarrollo de la planta, según mencionan Ma et al. (2014); Boogar \& Salehi (2020) y Chen et al. (2020): i) mínimo, se genera pérdida de turgencia celular, reducción de la tasa de expansión celular, disminución de la síntesis de pared celular y limitaciones en la síntesis de proteínas; ii) moderado, incide en aumentos del ácido abscísico (ABA) y cierre estomático parcial o total; y iii) máximo, en el que la planta produce cavitación de los elementos del xilema, caída de la hoja, acumulación de solutos orgánicos, hasta llegar al punto de marchitez de la planta. Conforme el periodo de exposición de estrés se aumente, la afectación a la planta se incrementa. Estudios desarrollados por
Miyazawa et al. (2014) mencionan que los daños en los procesos fisiológicos de especies tropicales pueden ser permanentes, si la exposición a estrés hídrico es prolongada, y afectar la turgencia foliar, traspiración y capacidad fotosintética de forma permanente.

Estudios desarrollados por Kaya et al. (2019) han determinado que periodos intermedios de sequía pueden generar daños en los procesos de fotosíntesis de las plantas, específicamente en el fotosistema II (PSII); en exposiciones cortas el daño puede ser temporal con reducción en la capacidad de fotosíntesis y genera problemas de adaptabilidad lumínica por parte de la planta. Con periodos de estrés hídrico prolongados, la afectación puede ser permanente, con incapacidad en desarrollo optimizado de la fotosíntesis e ineficiencia en los productos de los procesos de fotosíntesis, debido a procesos de fotooxidación y fotoinhibición que evitan que la planta desarrolle fotosíntesis en condiciones de déficit hídrico; esto, como estrategia de prevención de pérdida de turgencia foliar (Jiménez et al., 2010; Wakamori et al., 2020).

En conjunto con la variación de la capacidad fotosintética de las plantas, el estrés genera cambios en la reflectancia y fluorescencia de las plantas (Díaz-Barradas et al., 2020). Producto de la afectación del PSII, se dan procesos de oxidación de moléculas de clorofila, lo cual genera que las plantas estresadas reduzcan sus concentraciones de clorofila y, con ello, la capacidad fotosintética disminuya (Di Vaio et al., 2013; Ortuño et al., 2006). Además, el estrés genera el incremento en la actividad del ciclo de las xantofilas, el cual consiste en una disminución de grupos epoxi de las xantofilas de las plantas y formación de grupos del carotenoides que inciden en la coloración amarillenta de las hojas. El incremento del ciclo de las xantofilas es una evidencia de daños moderados del 
PSII y de la conservación de energía por parte de la planta (Holst et al., 2004), esto se evidencia en cambios en la reflectancia entre los 500 y 600 nm (Fournier \& Andrieu, 1998). A nivel de fluorescencia se generan cambios susceptibles en los indicadores de clorofila a, debido al incremento de las concentraciones de zeaxantina (Sánchez-Costa et al., 2015).

Otro de los efectos del estrés hídrico en las plantas se denota en la hidráulica foliar; estudios desarrollados por Valverde y Arias (2020), con especies tropicales, han determinado que el estrés hídrico genera pérdidas de turgencia foliar entre el 10 y $15 \%$, aspecto que incide en que el equilibrio entre la transpiración y absorción de agua de la planta se vea afectado y, por ende, la planta deba reducir la traspiración con cierre de estomas como medida de conservación hídrica, lo cual genera que la turgencia foliar se vea disminuida como mecanismo de reducción a la formación de embolismo en el tallo. Según Huzsvai \& Rajkai (2009), en especies tropicales es muy común, debido a la poca variabilidad de la temperatura en el año y periodos secos definidos en varias regiones del trópico, además repercute en que muchas especies reduzcan al máximo la turgencia con caída de hojas como mecanismo de preservación hídrica.

En el caso específico de Gmelina arborea Roxb, es una especie arbórea originaria del sureste asiático, se caracteriza por tener una alta tasa de crecimiento, adaptabilidad de suelos arcillosos, con deficiencias nutricionales menores y con buen sistema de drenaje (Tenorio et al., 2016). Es una especie ampliamente reforestada en Costa Rica, debido a su facilidad de adaptación a condiciones de plantaciones mixtas o puras (Adekunle et al., 2011) y su madera presenta excelentes propiedades físicas y mecánicas, lo cual permite que sea ampliamente utilizada en sectores como la construcción, mueblería y tarimas (Moya et al., 2017). El proceso de establecimiento de plantaciones de G. arborea es una de las etapas de mayor relevancia para el cultivo; factores ambientales como temperatura, precipitación, humedad relativa y viento pueden afectar la sobrevivencia de las platas al generar procesos de estrés hídrico (Eby et al., 2017). Sin embargo, son limitados los estudios disponibles que den caracterizaciones de la especie, que muestren su comportamiento en periodos de sequía moderados a extensos. Entre estos destaca el de Rojas et al. (2012), que analizó el comportamiento fisiológico y químico de plántulas, arboles jóvenes y maduros de G. arborea en condiciones de sequía y encontró que la transpiración disminuye conforme aumente la edad de la planta; las concentraciones de clorofila y carotenoides se incrementan conforme mayor sea la edad de la plántula.

Ante la ausencia de estudios sobre la capacidad de respuesta al estrés hídrico de la especie G. arborea, se estableció como objetivo caracterizar la fluorescencia, reflectancia y respuesta fisiológica al estrés hídrico en plántulas de Gmelina arborea Roxb, manteniendo la hipótesis de que el estrés hídrico genera una reducción fisiológica significativa en las plantas, lo cual se evidencia en cambios en los patrones de fotosíntesis, fluorescencia y reflectancia de la especie.

\section{METODOLOGÍA}

\section{Especie y condiciones del estudio}

Se seleccionaron 60 plántulas de Gmelina arborea, las cuales procedían de un mismo clon, esto con el fin de uniformizar las variables genéticas del experimento. Las plántulas se seleccionaron por tener la misma edad de seis semanas, tener una homogeneidad en cuanto altura, diámetro en 
la base y cantidad de hojas. El material procedió de una colección clonal especializada para la producción de madera comercial ubicada en Santa Clara, San Carlos Costa Rica (10²1'43,82” N y 84³0’38,30” O).

Las plántulas se plantaron en bandejas plásticas con compartimentos de 4,5 x 4,5 cm y $15 \mathrm{~cm}$ de profundidad; a las bandejas se les colocó suelo procedente de una finca productiva de Gmelina arbórea con fines maderables. El sustrato franco arcilloso, con un $\mathrm{pH}$ de 5,2, con niveles nutricionales de nitrógeno, fósforo y potasio óptimos para el crecimiento de la especie. Posterior a tres semanas de aclimatación, las bandejas de plántulas se trasladaron al sitio de estudio en Cartago, Costa Rica.

El estudio se desarrolló en el Laboratorio de Ecofisiología Forestal y Aplicaciones Ecosistémicas (ECOPLANT) del Tecnológico de Costa Rica (9॰50'57,91'” N; 8354'37,27' O), Cartago, Costa Rica. Situado a una altitud de $1392 \mathrm{~m}$, con una temperatura controlada promedio de $25^{\circ} \mathrm{C}$ y humedad relativa del 70 $\%$. Durante todo el experimento se controlaron las condiciones ambientales, la temperatura no varió más de $\pm 2{ }^{\circ} \mathrm{C}$ y la humedad relativa $\pm 10 \%$. El área de estudio presentó una taza de luz fotosintética activa homogénea de 20 $\mu \mathrm{mol} \mathrm{m} \mathrm{m}^{-2} \mathrm{~s}^{-1}$, con un fotoperiodo de 12 horas definido de las 05:00 a 17:00 (GMT-6 América Central). Antes del inicio del experimento las plántulas se aclimatizaron al sitio de estudio, para lo cual se les aplicó una dosis diaria de $20 \mathrm{ml}$ de agua por planta; el proceso fue automatizado, mediante una bomba automática con aspersores por goteo.

\section{Condiciones de estrés hídrico implementadas}

Se implementaron dos tratamientos: control (testigo) y estrés hídrico máximo. A las plantas testigo (30 plántulas) se les aplicó una dosis constante de agua durante todo el experimento, se les aplicaron $20 \mathrm{ml} \mathrm{de}$ agua cada dos días. En caso de las plantas con estrés hídrico máximo (30 plántulas), durante los primeros 5 días del experimento solo se les aplicaron $5 \mathrm{ml}$ de agua cada dos días; posterior a dicho periodo, las plantas no recibieron agua en el tiempo restante del experimento. El estudio se desarrolló con un diseño simple aleatorio.

\section{Acumulación de biomasa}

Se analizó la acumulación de biomasa tanto al inicio como final del experimento, para lo cual se cosecharon ocho plantas por tratamiento; cada individuo se seccionó en tallo, raíz y hojas, esto con el fin de analizar la biomasa de cada órgano por aparte. Seguidamente la muestra se secó por un periodo de 72 horas a $105^{\circ} \mathrm{C}$ y se pesó en condición seca para estimar la biomasa seca.

\section{Variables fisiológicas evaluadas}

A cada planta de G. arborea se le seleccionó una hoja de edad intermedia, libre de defectos (daños por patógenos, mecánicos producidos por manejo de las plantas o deformidades genéticas) para valorar los siguientes parámetros: tasa fotosintética neta (An), conductancia estomática (Gs), contenido relativo de clorofila (SPAD) y turgencia foliar (Pp). Las curvas de An se desarrollaron al inicio (día 0) y el día final de la prueba (día 31), mientras An máximo, Gs, SPAD y Pp se evaluaron cada siete días. Las mediciones se realizaron entre las 09:00 y 11:00 (GMT-6 Central América); se llevó control de la temperatura, humedad relativa y luminosidad, con el fin de verificar que su variabilidad no infiriera en los datos obtenidos. A continuación, se especifica el procedimiento realizado para cada parámetro: 
i. Tasa fotosintética neta (An): se utilizó un medidor de intercambio gaseoso CIRAS-II (PP Systems ${ }^{\circledR}$, USA). El CIRAS-II que se calibró con un flujo constante de $\mathrm{CO}_{2}$ de $380 \mathrm{ppm}$ y se programó para realizar las curvas de asimilación fotosintética de 0 a $2000 \mu \mathrm{mol}$ (quanta) $\mathrm{m}^{-2} \mathrm{~s}^{-1}$, en intervalos de $250 \mu \mathrm{mol}$ (quanta) $\mathrm{m}^{-2} \mathrm{~s}^{-1}$, en un área estándar de $2,5 \mathrm{~cm}^{2}$ de la hoja.

ii. Conductividad estomática: se implementó un Leaf Porometer CS-1 (Degacon Devices, Inc $\left.{ }^{\circledR}\right)$ que cuenta con una cámara de apertura de medición de 6,35 $\mathrm{mm}$ de diámetro.

iii. Valor del contenido relativo de clorofila en las hojas: las mediciones del contenido potencial de clorofila se realizaron con un SPAD-502 (Konica Minolta ${ }^{\circledR}$ ) que cuenta con un área efectiva de valoración de $5 \mathrm{~mm}^{2}$.

iv. Turgencia Foliar: se estimó mediante unidades ZIM-probe (Yara ${ }^{\circledR}$ ) de 82 $\mathrm{mm}^{2}$ de cobertura, programada para tomar los datos cada 15 minutos según recomienda la metodología de Zimmermann et al. (2008); los valores de turgencia foliar $(\mathrm{Pp})$ son generados a partir del principio de la ecuación 1 .

$$
P_{p}=\left(\frac{b}{a P_{c}+b}\right)^{\frac{1}{a}} \cdot F_{a} \cdot P_{c l a m p}
$$

Donde $a$ y $b$ son constantes, $\mathrm{F}_{\mathrm{a}}$ es el factor de atenuación específico de la hoja y $P_{\text {clamp }}$ es un factor de atenuación del sensor.

\section{Medición de reflectancia}

La evaluación hiperespectral, específicamente de la reflectancia, se desarrolló con un espectro radiómetro portable de PP System, tipo UniSpec SC. Se trabajó con un rango de 310 a $1100 \mathrm{~nm}$ (espectro visible e infrarrojo cercano), con un intervalo de medición de $10 \mathrm{~nm}$ y tiempo de medición programado de $4 \mathrm{~ms}$. El sensor implementado contó con una certificación estándar "SMA 905 conectors", acorde para este tipo de instrumentación, con un área efectiva de $3 \mathrm{~mm}^{2}$. El sensor se colocó perpendicular a la superficie mediante un compartimento cerrado, con el fin de evitar sesgos en la medición por efecto de la luz externa. Todas las mediciones se desarrollaron en un sitio con una luminosidad homogénea de 600 lux.

\section{Medición de fluorescencia}

Para la determinación de la fluorescencia se implementó un flurómetro modelo FluoroMax de quinta generación con una luz de $150 \mathrm{~W}$ de xenón libre de ozono, con un tiempo te integración de 0,1 segundos y con un rango de operabilidad del espectro de 185 a $850 \mathrm{~nm}$. Las hojas utilizadas en el fluorómetro se condicionaron en fase oscura 30 minutos antes del estudio, posterior a ello se realizó un muestreo en las áreas relacionadas a la clorofila a $(420$ y $690 \mathrm{~nm})$ y clorofila b (453 y $642 \mathrm{~nm}$ ).

\section{Análisis estadístico}

Se implementó un diseño experimental simple aleatorio, en el cual se evaluó, si existen diferencias significativas entre los tratamientos de estrés hídrico en cada fecha de medición, para lo cual se implementó un análisis de varianza de una vía (One way ANDEVA) y a las variables que mostraron diferencias se les aplicó prueba de Tukey con una significancia de 0,05 . Los análisis se realizaron con en el programa STATISTICA 9.0 y ORIGIN Pro 2020. En cada análisis se verificaron los supuestos de normalidad de los residuales y la homogenización de las varianzas. 
Tabla 1. Biomasa acumulada al inicio (día 0) y final (día 31) del experimento en plantas de G. arborea expuestas en condiciones de estrés hídrico

\begin{tabular}{cccc}
\hline & Segmento de la planta & Plantas testigo & Plantas en estrés hídrico \\
\hline & Foliar & $0,789^{\mathrm{a}}(0,023)$ & $0,719^{\mathrm{a}}(0,033)$ \\
Día 0 & Tallo & $0,188^{\mathrm{a}}(0,018)$ & $0,192^{\mathrm{a}}(0,010)$ \\
& Radicular & $0,274^{\mathrm{a}}(0,020)$ & $0,219^{\mathrm{a}}(0,011)$ \\
& Total & $1,251^{\mathrm{a}}(0,028)$ & $1,130^{\mathrm{a}}(0,028)$ \\
& Foliar & $1,100^{\mathrm{a}}(0,022)$ & $0,781^{\mathrm{b}}(0,043)$ \\
Día 31 & Tallo & $0,227^{\mathrm{a}}(0,028)$ & $0,190^{\mathrm{b}}(0,010)$ \\
& Radicular & $0,321^{\mathrm{a}}(0,034)$ & $0,233^{\mathrm{b}}(0,028)$ \\
& Total & $1,648^{\mathrm{a}}(0,089)$ & $1,204^{\mathrm{b}}(0,043)$ \\
\hline
\end{tabular}

Nota. Letras distintas muestran diferencias significativas al 0,05. Valores en paréntesis corresponden a desviación estándar.

\section{RESULTADOS}

\section{Acumulación de biomasa}

En la tabla 1 se presenta la acumulación de biomasa presente en plantas testigo y con estrés hídrico tanto al inicio (día 0) como final (día 31) del experimento. En el inicio del experimento no se encontraron diferencias significativas entre los tratamientos, con una biomasa total promedio de $1,190 \mathrm{~g}$, presente en un $62 \%$ en las hojas, $28 \%$ en la raíz y $10 \%$ en el tallo. Patrón que a los 31 días cambió, y las plantas testigo fueron las que mostraron una acumulación de biomasa significativamente mayor $(1,648$ g), en comparación con las plantas en estrés hídrico $(1,204 \mathrm{~g})$; el estancamiento de las plantas con estrés fue del 26, 9\% y las hojas el órgano mostraron la mayor diferenciación con las plantas testigo (del 29,1 $\%$, mientas que el tallo y raíz mostraron la menor diferenciación $(16,3 \% \mathrm{y}$ $27,4 \%$ respectivamente).

\section{Curvas fotosintéticas}

En la Figura 1 se presentan las curvas fotosintéticas de G. arborea al inicio y final del experimento. El comportamiento indiferentemente del día del experimento o el tratamiento fue el mismo, una curva logística, con un aumento de la An, conforme al PPFD se incrementó hasta llegar un punto de An máximo en donde se estabilizó la fotosíntesis. En el día cero la An máxima de la plantas llegó a ser de 9,56 $\mu \mathrm{mol} \mathrm{m} \mathrm{m}^{-2}$

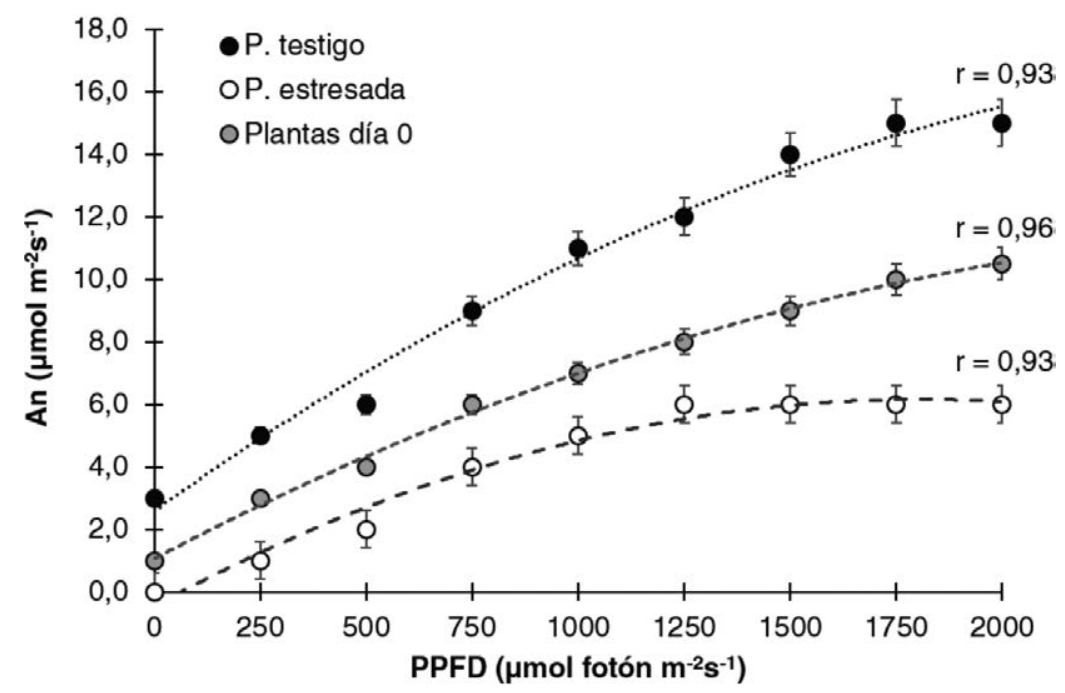

Figura 1. Curvas de asimilación fotosintética neta (An) con respecto a la densidad de flujo fotónico fotosintético (PPFD), obtenida en día 0 y día 31 en plantas de G. arborea expuestas a condiciones de estrés hídrico. 
$\mathrm{s}^{-1}$ con una estabilización de la curva a los $1750 \mu \mathrm{mol}$ fotón $\mathrm{m}^{-2} \mathrm{~s}^{-1} \mathrm{y}$ sin presentar diferencias entre los tratamientos,: este aspecto varió en el día 31; donde la An fue mayor en las plantas testigo, al obtener un An máximo de 14,6 $\mu \mathrm{mol} \mathrm{m}^{-2}$ $\mathrm{s}^{-1}$, con una estabilización de la curva a los $1500 \mu \mathrm{mol}$ fotón $\mathrm{m}^{-2}$ $\mathrm{s}^{-1}$. Mientras que las plantas con estrés hídrico presentaron una disminución de la capacidad fotosintética con un An máximo de $5,8 \mu \mathrm{mol} \mathrm{m}^{-2} \mathrm{~s}^{-1}$, con estabilización de la curva a los 1250 $\mu$ mol fotón $\mathrm{m}^{-2} \mathrm{~s}^{-1}$, lo cual significó una reducción del $40 \%$ de la capacidad fotosintética de las plantas en comparación al día 0 y del $60,1 \%$ con respecto a las plantas testigo en el mismo periodo de tiempo.

\section{Caracterización fisiológica}

Se determinó que en la asimilación fotosintética máxima (figura 2a), las plantas testigo presentaron un valor promedio de $12,6 \mu \mathrm{mol} \mathrm{m} \mathrm{m}^{-2} \mathrm{~s}^{-1}$ durante todo el experimento; mientras las plantas en estrés hídrico presentaron una disminución de An max durante los primeros 14 días del $52 \%$ y obtuvieron un valor de $5,4 \mu \mathrm{mol} \mathrm{m}^{-2} \mathrm{~s}^{-1}$; posterior del día 14 hasta el final del experimento la An max se mantuvo con pocas variaciones con un valor promedio de 5,6 $\mu \mathrm{mol}$ $\mathrm{m}^{-2} \mathrm{~s}^{-1}$. En el caso de la conductividad estomática (Figura 2b), las plantas testigo mostraron poca variación, con una conductancia promedio de $256 \mathrm{mmol} \mathrm{m}^{-2} \mathrm{~s}^{-1}$; en cambio las plantas con estrés hídrico en los primeros 14 días presentaron una reducción del $60 \%$ de la conductancia estomática, con un valor
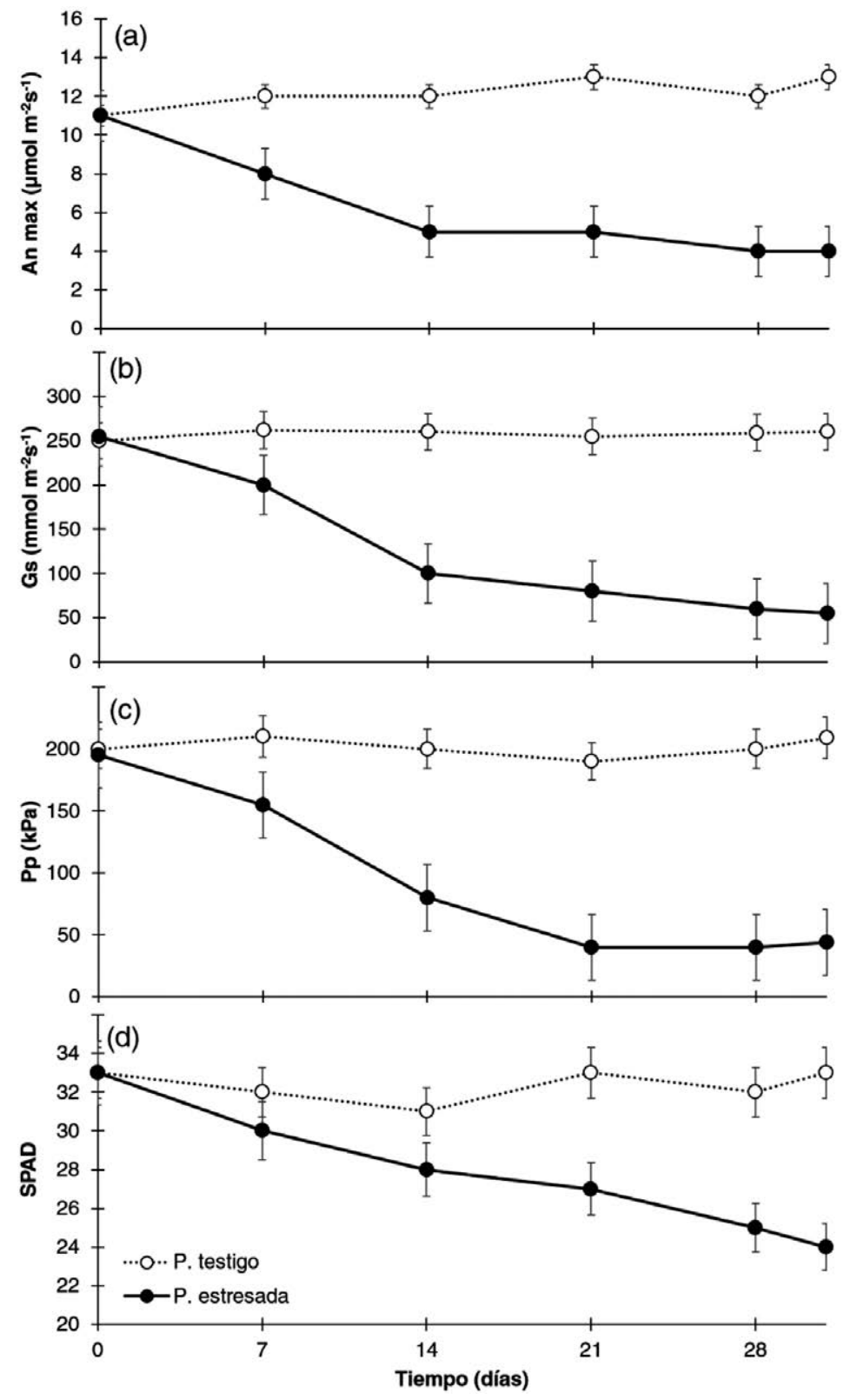

Figura 2. Variabilidad de la asimilación fotosintética máxima (An max), conductividad estomática (Gs), turgencia foliar (Pp) y contenido relativo de clorofila (SPAD) en función al tiempo en plantas de G. arborea expuestas a condiciones de estrés hídrico. Las barras de error corresponden a la desviación estándar. 

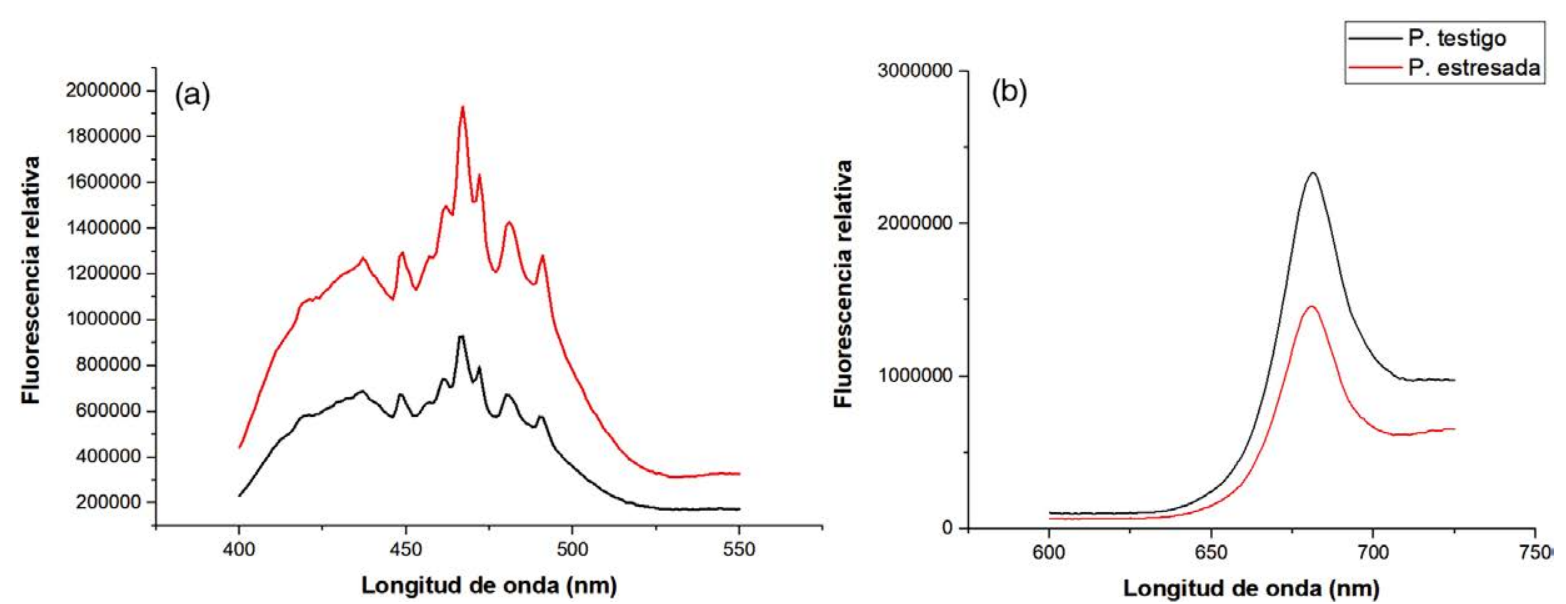

Figura 3. Fluorescencia de los 400 a $550 \mathrm{~nm}$ (a) y de 600 a $725 \mathrm{~nm}$ (b) en plántulas con distintas niveles en plantas de G. arborea expuestas a condiciones de estrés hídrico.

de $109 \mathrm{mmol} \mathrm{m} \mathrm{m}^{-2} \mathrm{~s}^{-1}$, comportamiento que se mantuvo con leves decrecimientos de la conductancia hasta el final del experimento que fue de $65 \mathrm{mmol} \mathrm{m}^{-2} \mathrm{~s}^{-1}$, un diferencial del $75 \%$ con respecto a la planta testigo.

En el caso de la turgencia foliar (figura 2c), posterior de siete días de exposición a estrés hídrico, se dieron diferencias significativas entre ambos tratamientos: en las plantas testigo la turgencia mantuvo un comportamiento homogéneo, con un valor promedio de $210 \mathrm{kPa}$ en los 31 días del experimento; caso distinto a las plantas con estrés hídrico que en los primeros 21 días presentaron una pérdida de la turgencia foliar en un $76 \%$, con valores promedio de 48 $\mathrm{kPa}$, es posterior al día 21 cuando se da una estabilización de la turgencia en los $45 \mathrm{kPa}$ hasta el final del experimento. Finalmente, con el contenido relativo de nitrógeno (Figura 2d) en plantas testigo el valor SPAD promedio fue de 32,6 el cual mostró poca variación en los 31 días; caso contrario a las plantas con estrés hídrico que presentaron un constante decrecimiento del valor SPAD conforme el tiempo de estrés hídrico se incrementó, alcanzó, al día 31, una diferencia del $26 \%$, con valores promedio de 23,7 .

\section{Análisis de la fluorescencia}

Con respecto a la fluorescencia (Figura 3), se determinó en el rango de 400 a $550 \mathrm{~nm}$. Las plantas con estrés hídrico presentaron un nivel de fluorescencia significativamente mayor en comparación con las plantas testigo, los puntos de diferenciación se identificaron a los $453 \mathrm{~nm}$ (punto se desarrolla la clorofila b), $474 \mathrm{~nm}, 485 \mathrm{~nm}$ y 494 nm (figura 3a), a diferencia en la florescencia entre ambos tratamientos fue del 15 a $32 \%$. En cambio, de los 600 a 725 nm (figura 3b), las plantas testigo fueron las que presentaron una fluorescencia significativamente mayor en comparación con las plantas estresadas, donde fue a los $682 \mathrm{~nm}$ el punto de mayor diferenciación de la fluorescencia y cercano al área de representación de la clorofila a (generalmente a los $690 \mathrm{~nm}$ ), la distancia de fluorescencia entre ambos tratamientos fue en promedio del $19 \%$.

\section{Análisis de reflectancia}

Se determinó la gráfica representativa de la reflectancia y la primera derivada de la reflectancia para las plantas testigo y en condición de estrés hídrico al día 31 
(Figura 4), de los 310 a $460 \mathrm{~nm}$ no se encontraron diferencias significativas entre ambos tratamientos (ver flechas en las figuras). Además, no se encontró una diferencia significativa entre los 775 y 1150 $\mathrm{nm}$, resultó similar el comportamiento de reflectancia entre ambos tratamientos. En el rango de los 460 a $770 \mathrm{~nm}$ se obtuvo una variación entre tratamientos (figura 4a): las plantas en condición de estrés hídrico presentaron una reflectancia significativamente mayor, del 10 al $30 \%$ superior en comparación con las testigo. Al analizar el rango con la primera derivada (Figura $4 b$ ), se obtuvieron dos puntos diferenciadores que fueron a los $490 \mathrm{~nm}$ y $700 \mathrm{~nm}$, puntos en los que se desarrolla la clorofila a, y fue la planta condición de estrés la que presentó la mayor excitación de reflectancia.

\section{DISCUSIÓN}

La reducción de biomasa obtenida en las plantas con estrés es la respuesta mecánica a la reducción hídrica como mecanismo de ahorro energético (Hsiao, 1931; Valverde y Arias, 2020), las plantas detienen el crecimiento de los meristemos cuando se producen desequilibrios entre la absorción y traspiración de agua, como un mecanismo de regulación (Pollastrini et al., 2010), en especial, se da una reducción en la formación de nuevas láminas foliares, debido al alto costo energético e hídrico que tienen para la planta. Estudios desarrollaros por Dell'Amico et al. (2012) muestran que el desarrollo de láminas foliares representan para la planta un $26 \%$ de su recurso hídrico y $20 \%$ de su recurso energético, por lo que su estancamiento es una medida inmediata de ahorro energético. El comportamiento obtenido es similar al reportado por Valverde y Arias (2020) con la especie Gliricidia sepium, pues encontraron, en 98 días, una diferencia del $60 \%$ de la biomasa entre plantas de control y con estrés lineal, y la reducción foliar fue de un $70 \%$. Esto se argumentó como la respuesta de la planta a la producción de $\mathrm{ABA}$, como mecanismos que retardan el crecimiento, reducen la cobertura foliar y promueve solutos orgánicos en las hojas, con el fin de mitigar la pérdida hídrica en la planta (Bader et al., 2015).
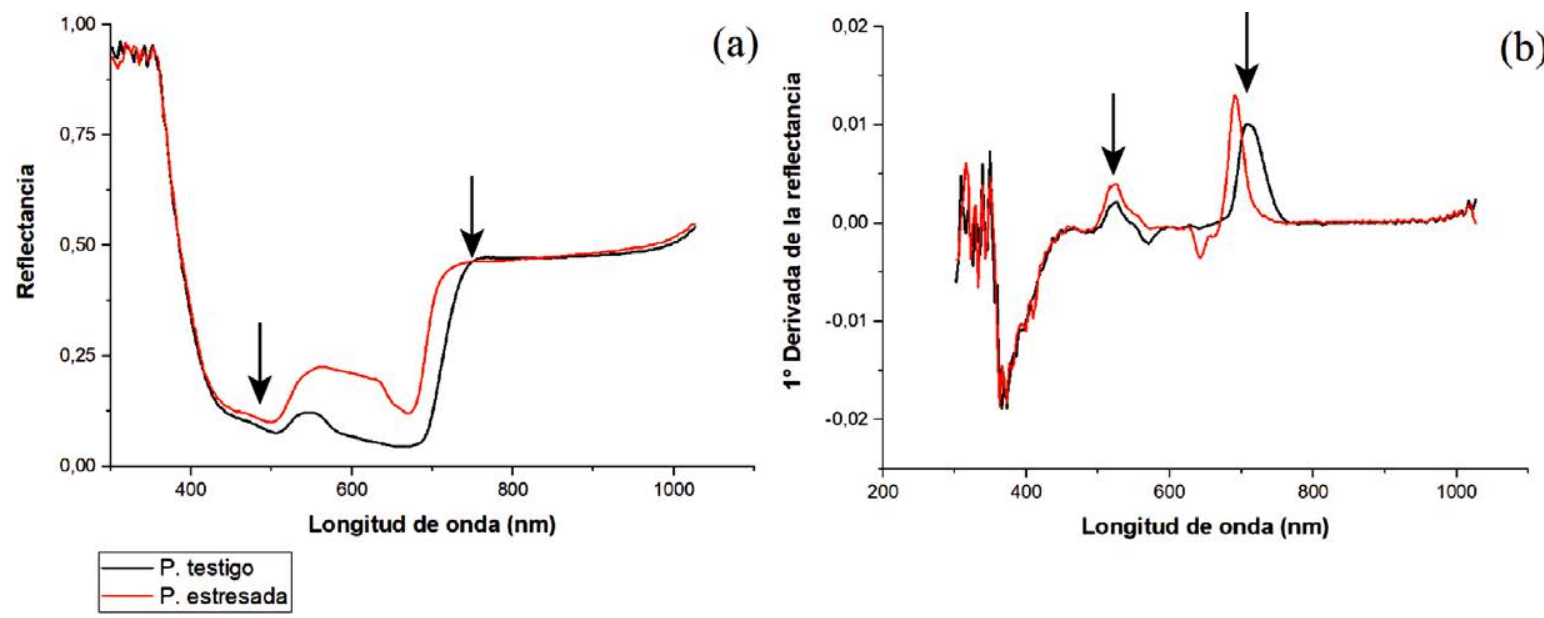

Figura 4. Reflectancia (a) y primera derivada (b) de la reflectancia de los 310 a $1150 \mathrm{~nm}$ en plantas de G. arborea expuestas a condiciones de estrés hídrico. 
Otro aspecto que se evidenció en las plantas con estrés fue la reducción de la fotosíntesis; como resultado de la menor disponibilidad de agua en el sistema, las plantas comienzan a cerrar estomas, lo que afecta la entrada de $\mathrm{CO}_{2}$ y desarrollo de la fotosíntesis. Boogar \& Salehi (2020) mencionan que es un parámetro altamente sensible en especies tropicales, que se puede ver disminuido en menos de una semana de estrés moderado. La reducción de transpiración y disminución de los procesos metabólicos de la planta inciden en que la fotosíntesis se reduzca, aspecto que Paulus et al. (2014) mencionan que puede ser promotor de procesos de daños fotofoliares en la planta, debido a la acumulación de solutos orgánicos, diminución de intercambio gaseoso y calentamiento de la lámina foliar, lo cual incide en daños del PSII y PSI (fotosistema I). Eby et al. (2017) mencionan que la fotosíntesis, al verse reducida afecta directamente la capacidad de formación de azúcares y encimas primarias para la planta, incidiendo, en una menor capacidad de recuperación del embolismo o capacidad de respuesta a daños mecánicos.

La afectación de la fotosíntesis se vincula con el cierre de estomas (en caso del estudio, superior al $60 \%$ ), pérdida de clorofila y turgencia foliar. Maatallah et al. (2010) indican que el cierre de estomas es una acción inmediata a la pérdida de turgencia en las hojas cuando hay déficit moderado o severo en la planta. Al no tener suficiente agua, la lámina foliar tiende a perder humedad, lo cual afecta su angulación con respecto al sol y su espesor, aspectos que inciden en que el organismo tienda a cerrar estomas para aumentar su regulación y permitir que la planta pueda recuperar la turgencia en el corto plazo. Agam et al. (2013) mencionan que en especies tropicales el cambio de angulación de las hojas, producto de la reducción de la turgencia, tiende a ser una estrategia de reducción de la fotosíntesis y de disipación calórica; esto, debido que la hoja, al tener menos agua, le será más difícil disipar el calor, lo que resulta en daños a las células de la hoja. Ello puede evidenciarse en aperturas involuntarias de estomas, disminución de la resistencia estomática e inestabilidad fotosintética (De la Rosa et al., 2015).

Las reducciones de las variables fisiológicas inciden en cambios en la fluorescencia y reflectancia de las hojas; en el caso de la fluorescencia los daños generados en el PSII, la reducción de la clorofila producto de la oxidación de moléculas y el aumento del ciclo de las xantofilas (Vilfan et al., 2016) repercutieron en que la concentración de la clorofila a y b cambiara, aspecto que generó que la fluorescencia en el rango de la clorofila a aumentara y en el rango de la clorofila $b$ disminuyera. Esto, debido a que la clorofila a es susceptible al calor y su degradación es más simple en comparación a la clorofila b (Li et al., 2020), aspecto que incidió en que con mayor facilidad se aumentara la fluorescencia con menor intensidad de luz, lo cual Zhu et al. (2015) mencionan como producto de la fotoxidación y fotorreceptibilidad de la planta.

En el caso de la reflectancia, el cambio obtenido de los 500 y $600 \mathrm{~nm}$ se debe al amarillamiento de las hojas producto a la disminución de grupos epoxi de las xantofilas de las plantas y formación de grupos del carotenoides (Sonobe et al., 2018). En conjunto con los daños generados al PSII, los que inciden en que la planta no tenga el mismo patrón de reflectancia por la oxidación de la clorofila y variación de la concentración de la clorofila a y b (Naumann et al., 2008). Marín-Ortiz et al. (2020) mencionan se pueden dar incrementos de 
reflectancia entre el 15 y $25 \%$ en plantas con estrés hídrico elevado, por las variaciones de clorofila y efectos de la reducción de la turgencia foliar, que afecta en un calentamiento de la lámina foliar, lo que genera daños en las estructuras vegetales, en las cuales, en muchos casos, se evidencia un aclaramiento de la lámina foliar y aumento de su reflectancia.

\section{CONCLUSIONES}

La biomasa en plantas con estrés se redujo en un $26,9 \%$ y fue la cobertura foliar la que mostró mayor afectación (promedio de $29,1 \%)$. Con respecto a la fotosíntesis, la reducción fue del $60,1 \%\left(5,8 \mu \mathrm{mol} \mathrm{m}^{-2}\right.$ $\left.\mathrm{s}^{-1}\right)$ en relación con la planta testigo $(14,6$ $\mu \mathrm{mol} \mathrm{m} \mathrm{m}^{-2} \mathrm{~s}^{-1}$. Con la conductancia estomática, turgencia y contenido relativo de clorofila se presentó una disminución de sus valores en el tiempo, con una contracción del 20 al $60 \%$. En el caso de la fluorescencia, se determinaron dos patrones: de los 400 a $550 \mathrm{~nm}$ la fluorescencia se incrementó en las plantas estresadas; mientras de los 600 a $725 \mathrm{~nm}$ fueron las plantas testigo las que presentaron mayor excitación, en ambos rangos se presentaron valores máximos en los puntos correspondientes a clorofila a y b. Finalmente, la reflectancia mostró una diferenciación entre los $460 \mathrm{y}$ $770 \mathrm{~nm}$ y las plantas en condiciones de estrés resultaron ser las que presentaron una reflectancia mayor.

\section{RECONOCIMIENTOS}

Se agradece a la Vicerrectoría de Investigación y Extensión y la Laboratorio de Ecofisiología Forestal y aplicaciones ecosistémicas (ECOPLANT) del Tecnológico de Costa Rica por el apoyo financiero, equipos y tiempo para el desarrollo del experimento; también a Genforest por la disposición de los clones empleados en el experimento.

\section{DECLARACIÓN DE LA CONTRIBUCIÓN DE LOS AUTORES}

Se reconoce una distribución de la participación de la publicación de la siguiente manera: J.C.V. $40 \%$, D.A. $20 \%$, E.M. $20 \%$ y D.G. $20 \%$, J.C.V. desarrolló las mediciones fisiológicas en el experimento y análisis estadísticos del trabajo, D.A. desarrolló el diseño experimental, E.M. y D.G. participaron en las mediciones de reflectancia del estudio. Todos los autores colaboraron en la redacción y correcciones del manuscrito.

\section{DECLARACIÓN DE DISPO- NIBILIDAD DE LOS DATOS}

Los datos científicos que respaldan el estudio serán puestos a disposición por el autor J.C.V, los datos estarán disponibles mediante solicitudes específicas por parte del interesado vía dirección electrónica que se encuentra inscrita en el manuscrito.

\section{REFERENCIAS}

Adekunle, V., Alo, A. A., \& Adekayode, F. O. (2011). Yields and nutrient pools in soils cultivated with Tectona grandis and Gmelina arborea in Nigerian rainforest ecosystem. Journal of the Saudi Society of Agricultural Sciences, 10(2), 127-135. https://doi.org/10.1016/j. jssas.2011.05.001

Agam, N., Cohen, Y., Berni, J., Alchanatis, V., Kool, D., Dag, A., Yermiyahu, U., \& Ben-Gal, A. (2013). An insight to the performance of crop water stress index for olive trees. Agricultural Water Management, 118, 79-86. https://doi. org/10.1016/j.agwat.2012.12.004 
Bader, B., Aissaoui, F., Kmicha, I., Salem, A., Chehab, H., Gargouri, K., Boujnah, D., \& Chaieb, M. (2015). Effects of salinity stress on water desalination, olive tree (Olea europaea L. cvs "Picholine", "Meski" and 'Ascolana') growth and ion accumulation. Desalination, 364, 46-52. https://doi.org/10.1016/j. desal.2015.01.002

Beyer, R., Letort, V., \& Cournède, P. H. (2014). Modeling tree crown dynamics with 3D partial differential equations. Frontiers in Plant Science, 5, 1-8. https://doi.org/10.3389/ fpls.2014.00329

Boogar, A. R., \& Salehi, H. (2020). Developmental indices of cones collected from male plants of Juniperus polycarpos K. Koch under in vitro water deficit and salt stress conditions. South African Journal of Botany, 131, 277-282. https://doi.org/10.1016/j.sajb.2020.02.032

Chen, X., Zhao, P., Ouyang, L., Zhu, L., Ni, G., \& Schäfer, K. V. R. (2020). Whole-plant water hydraulic integrity to predict drought-induced Eucalyptus urophylla mortality under drought stress. Forest Ecology and Management, 468. https://doi.org/10.1016/j.foreco.2020.118179

De la Rosa, J. M., Domingo, R., Gómez-Montiel, J., \& Pérez-Pastor, A. (2015). Implementing deficit irrigation scheduling through plant water stress indicators in early nectarine trees. Agricultural Water Management, 152, 207-216. https://doi.org/10.1016/j.agwat.2015.01.018

Dell'Amico, J., Moriana, A., Corell, M., Girón, I., Morales, D., Torrecillas, A., \& Moreno, F. (2012). Low water stress conditions in table olive trees (Olea europaea L.) during pit hardening produced a different response of fruit and leaf water relations. Agricultural Water Management, 114, 11-17. https://doi. org/10.1016/j.agwat.2012.06.004

Di Vaio, C., Marallo, N., Marino, G., \& Caruso, T. (2013). Effect of water stress on dry matter accumulation and partitioning in pot-grown olive trees (cv Leccino and Racioppella). Scientia Horticulturae, 164, 172-177. https:// doi.org/10.1016/j.scienta.2013.09.008

Díaz-Barradas, M. C., Gallego-Fernández, J. B.; \& Zunzunegui, M. (2020). Plant response to water stress of native and non-native Oenothera drummondii populations. Plant Physiology and Biochemistry, 154, 219-228. https://doi. org/10.1016/j.plaphy.2020.06.001
Eby, W. M., Oyamakin, S. O.; \& Chukwu, A. U (2017). A new nonlinear model applied to the height-DBH relationship in Gmelina arborea. Forest Ecology and Management, 397, 139-149. https://doi.org/10.1016/j. foreco.2017.04.015

Farooq, M., Wahid, A., Kobayashi, N., Fujita, D., \& Basra, S. M. A. (2009). Plant drought stress: Effects, mechanisms and management. Sustainable Agriculture, 153-188. https://doi. org/10.1007/978-90-481-2666-8_12

Fournier, C., \& Andrieu, B. (1998). A 3̄ ral and process-based model of maize development. Annals of Botany, 81(2), 233-250. https://doi.org/10.1006/anbo.1997.0549

Gautier, H., Měch, R., Prusinkiewicz, P., \& Varlet-Grancher, C. (2000). 3D architectural modelling of aerial photomorphogenesis in white clover (Trifolium repens L.) using L-systems. Annals of Botany, 85(3), 359-370. https://doi. org/10.1006/anbo.1999.1069

Holst, T., Hauser, S., Kirchgäßner, A., Matzarakis, A., Mayer, H., \& Schindler, D. (2004). Measuring and modelling plant area index in beech stands. International Journal of Biometeorology, 48(4), 192-201. https://doi. org/10.1007/s00484-004-0201-y

Hsiao, T. C. (1931). Plant responses to sawdust. Proceedings of the Indiana Academy of Science, 41, 125-126.

Huzsvai, L., \& Rajkai, K. (2009). Modeling of plant adaptation to climatic drought induced water deficit. Biologia, 64(3), 536-540. https://doi. org/10.2478/s11756-009-0092-9

Jiménez, E., Vega, J. A., Pérez-Gorostiaga, P., Fonturbel, T., \& Fernández, C. (2010). Evaluation of sap flow density of acacia melanoxylon $\mathrm{R}$. Br. (blackwood) trees in overstocked stands in north-western Iberian Peninsula. European Journal of Forest Research, 129(1), 61-72. https://doi.org/10.1007/s10342-008-0252-4

Kaya, C., Ashraf, M., Wijaya, L., \& Ahmad, P. (2019). The putative role of endogenous nitric oxide in brassinosteroid-induced antioxidant defence system in pepper (Capsicum annuum L.) plants under water stress. Plant Physiology and Biochemistry, 143, 119-128. https://doi.org/10.1016/j.plaphy.2019.08.024

Li, W., Jiang, Y., Dong, M., Du, E., Zhou, Z., Zhao, S., \& Xu, H. (2020). Diverse responses of radial growth to climate across the southern part of the Asian boreal forests in northeast China. 
Forest Ecology and Management, 458(19). https://doi.org/10.1016/j.foreco.2019.117759

Ma, H., Song, J., Wang, J., Xiao, Z., \& Fu, Z. (2014). Improvement of spatially continuous forest LAI retrieval by integration of discrete airborne LiDAR and remote sensing multi-angle optical data. Agricultural and Forest Meteorology, 189-190, 60-70. https:// doi.org/10.1016/j.agrformet.2014.01.009

Maatallah, S., Ghanem, M. E., Albouchi, A., Bizid, E., \& Lutts, S. (2010). A greenhouse investigation of responses to different water stress regimes of Laurus nobilis trees from two climatic regions. Journal of Arid Environments, 74(3), 327-337. https://doi.org/10.1016/j.jaridenv.2009.09.008

Marín-Ortiz, J. C., Gutiérrez-Toro, N., Botero-Fernández, V., \& Hoyos-Carvajal, L. M. (2020). Linking physiological parameters with visible/near-infrared leaf reflectance in the incubation period of vascular wilt disease. Saudi Journal of Biological Sciences, 27(1), 88-99. https://doi.org/10.1016/j. sjbs.2019.05.007

Miyazawa, Y., Tateishi, M., Komatsu, H., Iwanaga, F., Mizoue, N., Ma, V., Sokh, H., \& Kumagai, T. (2014). Implications of leaf-scale physiology for whole tree transpiration under seasonal flooding and drought in central Cambodia. Agricultural and Forest Meteorology, 198, 221-231. https://doi.org/10.1016/j.agrformet.2014.08.013

Moreno, L. (2009). Respuesta de las plantas al estrés por déficit hídrico. Una revisión Plant responses to water deficit stress . A review. Agronomía Colombiana, 27(2), 179-191.

Moya, R., Rodríguez-Zúñiga, A., \& Puente-Urbina, A. (2017). Thermogravimetric and devolatilisation analysis for five plantation species: Effect of extractives, ash compositions, chemical compositions and energy parameters. Thermochimica Acta, 647, 36-46. https:// doi.org/10.1016/j.tca.2016.11.014

Naumann, J. C., Young, D. R., \& Anderson, J. E. (2008). Leaf chlorophyll fluorescence, reflectance, and physiological response to freshwater and saltwater flooding in the evergreen shrub, Myrica cerifera. Environmental and Experimental Botany, 63(13), 402-409. https://doi.org/10.1016/j. envexpbot.2007.12.008
Ortuño, M. F., García-Orellana, Y., Conejero, W., Ruiz-Sánchez, M. C., Alarcón, J. J., \& Torrecillas, A. (2006). Stem and leaf water potentials, gas exchange, sap flow, and trunk diameter fluctuations for detecting water stress in lemon trees. Trees - Structure and Function, 20(1), 1-8. https://doi.org/10.1007/ s00468-005-0004-8

Paulus, S., Schumann, H., Kuhlmann, H., \& Léon, J. (2014). High-precision laser scanning system for capturing 3D plant architecture and analysing growth ofcereal plants. Biosystems Engineering, 121, 1-11. https://doi.org/10.1016/j. biosystemseng.2014.01.010

Pollastrini, M., Desotgiu, R., Cascio, C., Bussotti, F., Cherubini, P., Saurer, M., Gerosa, G., \& Marzuoli, R. (2010). Growth and physiological responses to ozone and mild drought stress of tree species with different ecological requirements. Trees - Structure and Function, 24(4), 695-704. https://doi.org/10.1007/ s00468-010-0439-4

Rojas, A., Moreno, L., Melgarejo, L., \& Rodríguez, M. (2012). Physiological response of gmelina (Gmelina arborea Roxb.) to hydric conditions of the colombian Caribbean. Agronomía Colombiana, 30, 52-58.

Sánchez-Costa, E., Poyatos, R., \& Sabaté, S. (2015). Contrasting growth and water use strategies in four co-occurring Mediterranean tree species revealed by concurrent measurements of sap flow and stem diameter variations. Agricultural and Forest Meteorology, 207, 24-37. https://doi.org/10.1016/j. agrformet.2015.03.012

Sonobe, R., Sano, T., \& Horie, H. (2018). Using spectral reflectance to estimate leaf chlorophyll content of tea with shading treatments. Biosystems Engineering, 175, 168-182. https://doi. org/10.1016/j.biosystemseng.2018.09.018

Tenorio, C., Moya, R., Arias-Aguilar, D., \& Briceño-Elizondo, E. (2016). Biomass yield and energy potential of short-rotation energy plantations of Gmelina arborea one year old in Costa Rica. Industrial Crops and Products, 82, 63-73. https://doi.org/10.1016/j. indcrop.2015.12.005

Valverde, J. C., \& Arias, D. (2020). Efectos del estrés hídrico en crecimiento y desarrollo fisiológico de Gliricidia sepium (Jacq.) Kunth ex Walp. Colombia Forestal, 23(1), 29-53. https://doi. org/10.14483/2256201X.14786 
Varone, L., Ribas-Carbo, M., Cardona, C., Gallé, A., Medrano, H., Gratani, L., \& Flexas, J. (2012). Stomatal and non-stomatal limitations to photosynthesis in seedlings and saplings of Mediterranean species pre-conditioned and aged in nurseries: Different response to water stress. Environmental and Experimental Botany, 75, 235-247. https://doi.org/10.1016/j. envexpbot.2011.07.007

Vilfan, N., Vander Tol, C., Muller, O., Rascher, U., \& Verhoef, W. (2016). Fluspect-B: A model for leaf fluorescence, reflectance and transmittance spectra. Remote Sensing of Environment, 186, 596-615. https://doi.org/10.1016/j. rse.2016.09.017
Wakamori, K., Mizuno, R., Nakanishi, G., \& Mineno, H. (2020). Multimodal neural network with clustering-based drop for estimating plant water stress. Computers and Electronics in Agriculture, 168, 105118. https://doi. org/10.1016/j.compag.2019.105118

Zhu, L. W., Zhao, P., Wang, Q., Ni, G. Y., Niu, J. F., Zhao, X. H., Zhang, Z. Z., Zhao, P. Q., Gao, J. G., Huang, Y. Q., Gu, D. X., \& Zhang, Z. F. (2015). Stomatal and hydraulic conductance and water use in a eucalypt plantation in Guangxi, southern China. Agricultural and Forest Meteorology, 202, 61-68. https://doi. org/10.1016/j.agrformet.2014.12.003

\section{(c) $(i)$}

Fluorescencia, reflectancia y respuesta fisiológica al estrés hídrico en plántulas de Gmelina arborea Roxb (Juan Carlos Valverde • Dagoberto Arias-Aguilar • Ernesto Montero-Zeledón • Dionisio Gutiérrez-Fallas) Uniciencia is protected by Attribution-NonCommercial-NoDerivs 3.0 Unported (CC BY-NC-ND 3.0) 